\title{
Analytic study on two nonlinear evolution equations by using the $\left(G^{\prime} / G\right)$-expansion method
}

\author{
İsmail Aslan ${ }^{\mathrm{a}, *}$, Turgut Öziş ${ }^{\mathrm{b}}$ \\ a Department of Mathematics, Izmir Institute of Technology, 35430 Gulbahce, Urla, Izmir, Turkey \\ ${ }^{\mathrm{b}}$ Department of Mathematics, Ege University, 35100 Bornova, Izmir, Turkey
}

\section{A R T I C L E I N F O}

\section{Keywords:}

$\left(G^{\prime} \mid G\right)$-expansion method

Modified Camassa-Holm equation

Two-dimensional Korteweg-de-Vries-

Burgers equation

Exact solutions

Traveling wave solutions

\begin{abstract}
A B S T R A C T
The validity and reliability of the so-called $\left(G^{\prime} / G\right)$-expansion method is tested by applying it to two nonlinear evolutionary equations. Solutions in more general forms are obtained. When the parameters are taken as special values, it is observed that the previously known solutions can be recovered. New rational function solutions are also presented. Being concise and less restrictive, the method can be applied to many nonlinear partial differential equations.
\end{abstract}

(c) 2008 Elsevier Inc. All rights reserved.

\section{Introduction}

In the nonlinear sciences, it is well known that many nonlinear partial differential equations (PDEs) are widely used to describe the complex phenomena. So, the powerful and efficient methods to find analytic solutions of nonlinear equations have drawn a lot of interest by a diverse group of scientists, for example, Painleve expansion method [1], Jacobi elliptic function method [2], Hirota's bilinear method [3], the Sine-Cosine function method [4], the Tanh-Coth function method [5], the Exp-function method [6] and so on.

Recently, Wang et al. [7] proposed the $\left(G^{\prime} / G\right)$-expansion method and showed that it is powerful for finding analytic solutions of PDEs. Next, Bekir [8] applied the method to some nonlinear evolution equations gaining traveling wave solutions. Later, Zhang et al. [9] have generalized the method to obtain non-traveling wave solutions and coefficient function solutions and Zhang et al. [10] further extended the method to solve an evolution equation with variable coefficients.

In this paper, we choose the modified Camassa-Holm $(\mathrm{mCH})$ equation and the two-dimensional Korteweg-de-Vries-Burgers (2D-KdVB) equation to illustrate the $\left(G^{\prime} / G\right)$-expansion method. In the literature, researchers have obtained explicit exact solutions to the $\mathrm{mCH}$ equation independently by various methods. To mention, Wazwaz [11,12] successfully examined solitary wave solutions to the $\mathrm{mCH}$ equation by means of the tanh method, the sine-cosine method, the extended tanh method, etc. Elcin [13] studied the $\mathrm{mCH}$ equation using the Exp-function method and revealed new solutions. There are many studies for the 2D-KdVB equation, and some profound results have been established by the authors [14-20] using the well-known techniques; the extended tanh method, the homotopy perturbation method, the Adomian decomposition method, the first integral method, etc.

\section{Description of the $\left(G^{\prime} / G\right)$-expansion method}

We suppose that the given nonlinear partial differential equation for $u(x, t)$ to be in the form

$$
P\left(u, u_{x}, u_{t}, u_{x x}, u_{x t}, u_{t t}, \ldots\right)=0
$$

\footnotetext{
* Corresponding author.

E-mail address: ismailaslan@iyte.edu.tr (İ. Aslan).
} 
where $P$ is a polynomial in its arguments. The essence of the $\left(G^{\prime} / G\right)$-expansion method can be presented in the following steps:

Step 1. Seek traveling wave solutions of Eq. (1) by taking $u(x, t)=U(\xi), \xi=x-c t$, and transform Eq. (1) to the ordinary differential equation

$Q\left(U, U^{\prime}, U^{\prime \prime}, \ldots\right)=0$,

where prime denotes the derivative with respect to $\xi$.

Step 2. If possible, integrate Eq. (2) term by term one or more times. This yields constant(s) of integration. For simplicity, the integration constant(s) can be set to zero.

Step 3. Introduce the solution $U(\xi)$ of Eq. (2) in the finite series form

$U(\xi)=\sum_{i=0}^{N} a_{i}\left(\frac{G^{\prime}(\xi)}{G(\xi)}\right)^{i}$,

where $a_{i}$ are real constants with $a_{N} \neq 0$ to be determined, $N$ is a positive integer to be determined. The function $G(\xi)$ is the solution of the auxiliary linear ordinary differential equation

$G^{\prime \prime}(\xi)+\lambda G^{\prime}(\xi)+\mu G(\xi)=0$,

where $\lambda$ and $\mu$ are real constants to be determined.

Step 4. Determine $N$. This, usually, can be accomplished by balancing the linear term(s) of highest order with the highest order nonlinear term(s) in Eq. (2).

Step 5. Substituting (3) together with (4) into Eq. (2) yields an algebraic equation involving powers of $\left(G^{\prime} / G\right)$. Equating the coefficients of each power of $\left(G^{\prime} / G\right)$ to zero gives a system of algebraic equations for $a_{i}, \lambda, \mu$ and $c$. Then, we solve the system with the aid of a computer algebra system (CAS), such as Mathematica, to determine these constants. On the other hand, depending on the sign of the discriminant $\Delta=\lambda^{2}-4 \mu$, the solutions of Eq. (4) are well known to us. So, as a final step, we can obtain exact solutions of the given Eq. (1).

\section{Applications}

In this section, we will demonstrate the $\left(G^{\prime} / G\right)$-expansion method on two of the well-known nonlinear evolution equations, namely, the $\mathrm{mCH}$ equation and the 2D-KdVB equation.

\subsection{The $\mathrm{mCH}$ equation}

Let us consider the following celebrated $\mathrm{mCH}$ equation in the form:

$$
u_{t}-u_{x x t}+3 u^{2} u_{x}=2 u_{x} u_{x x}+u u_{x x x}
$$

Eq. (5) models shallow water waves, and is well known to be integrable, possessing multi-soliton solutions with peaks, socalled multi-peakons, see [11-13] and references therein. To look for the traveling wave solution of Eq. (5), we make the transformation $u(x, t)=U(\xi), \xi=x-c t$, where $c$ is the wave speed. Then, after integrating the obtained ODE once and setting the integration constant to zero, we get

$$
c\left(U^{\prime \prime}-U\right)+U^{3}-U U^{\prime \prime}-\frac{1}{2}\left(U^{\prime}\right)^{2}=0
$$

where prime denotes the derivative with respect to $\xi$. Now, we make an ansatz (3) for the solution of Eq. (6). Balancing the terms $U^{3}$ and $U U^{\prime \prime}$ in Eq. (6) yields the leading order $N=2$. Therefore, we can write the solution of Eq. (6) in the form

$$
U=a_{0}+a_{1}\left(\frac{G^{\prime}}{G}\right)+a_{2}\left(\frac{G^{\prime}}{G}\right)^{2} .
$$

By (4) and (7) we derive that

$$
\begin{aligned}
U^{\prime}(\xi)= & -2 a_{2}\left(\frac{G^{\prime}}{G}\right)^{3}-\left(a_{1}+2 a_{2} \lambda\right)\left(\frac{G^{\prime}}{G}\right)^{2}-\left(a_{1} \lambda+2 a_{2} \mu\right)\left(\frac{G^{\prime}}{G}\right)-a_{1} \mu \\
U^{\prime \prime}(\xi)= & 6 a_{2}\left(\frac{G^{\prime}}{G}\right)^{4}+\left(2 a_{1}+10 a_{2} \lambda\right)\left(\frac{G^{\prime}}{G}\right)^{3}+\left(8 a_{2} \mu+3 a_{1} \lambda+4 a_{2} \lambda^{2}\right)\left(\frac{G^{\prime}}{G}\right)^{2} \\
& +\left(6 a_{2} \lambda \mu+2 a_{1} \mu+a_{1} \lambda^{2}\right)\left(\frac{G^{\prime}}{G}\right)+2 a_{2} \mu^{2}+a_{1} \lambda \mu .
\end{aligned}
$$


Substituting (7)-(9) into (6), setting the coefficients of $\left(G^{\prime}(\xi) / G(\xi)\right)^{i}(i=0,1, \ldots, 6)$ to zero, we obtain the following underdetermined system of algebraic equations for $a_{0}, a_{1}, a_{2}, c, \lambda$ and $\mu$ :

$$
\begin{aligned}
& \left(\frac{G^{\prime}}{G}\right)^{0}:-c a_{0}+a_{0}^{3}+c \lambda \mu a_{1}-\lambda \mu a_{0} a_{1}-\frac{1}{2} \mu^{2} a_{1}^{2}+2 c \mu^{2} a_{2}-2 \mu^{2} a_{0} a_{2}=0, \\
& \left(\frac{G^{\prime}}{G}\right)^{1}:-c a_{1}+c \lambda^{2} a_{1}+2 c \mu a_{1}-\lambda^{2} a_{0} a_{1}-2 \mu a_{0} a_{1}-3 a_{0}^{2} a_{1}-2 \lambda \mu a_{1}^{2}+6 c \lambda \mu a_{2}-6 \lambda \mu a_{0} a_{2}-4 \mu^{2} a_{1} a_{2}=0, \\
& \left(\frac{G^{\prime}}{G}\right)^{2}: 3 c \lambda a_{1}-3 \lambda a_{0} a_{1}-\frac{3}{2} \lambda^{2} a_{1}^{2}-3 \mu a_{1}^{2}+3 a_{0} a_{1}^{2}-c a_{2}+4 c \lambda^{2} a l 2+8 c \mu a_{2}-4 \lambda^{2} a_{0} a_{2} \\
& \quad-8 \mu a_{0} a_{2}+3 a_{0}^{2} a_{2}-11 \lambda \mu a_{1} a_{2}-4 \mu^{2} a_{2}^{2}=0, \\
& \left(\frac{G^{\prime}}{G}\right)^{3}: 2 c a_{1}-2 a_{0} a_{1}-4 \lambda a_{1}^{2}+a_{1}^{3}+10 c \lambda a_{2}-7 \lambda^{2} a_{1} a_{2}-14 \mu a_{1} a_{2}+6 a_{0} a_{1} a_{2}-10 \lambda \mu a_{2}^{2}=0, \\
& \left(\frac{G^{\prime}}{G}\right)^{4}:-\frac{5}{2} a_{1}^{2}+6 c a_{2}-6 a_{0} a_{2}-17 \lambda a_{1} a_{2}+3 a_{1}^{2} a_{2}-6 \lambda^{2} a_{2}^{2}-12 \mu a_{2}^{2}+3 a_{0} a_{2}^{2}=0, \\
& \left(\frac{G^{\prime}}{G}\right)^{5}:-10 a_{1} a_{2}-14 \lambda a_{2}^{2}+3 a_{1} a_{2}^{2}=0, \\
& \left(\frac{G^{\prime}}{G}\right)^{6}:-8 a_{2}^{2}+a_{2}^{3}=0 .
\end{aligned}
$$

Solving the above system with the aid of Mathematica, we have the following three sets of solutions:

First solution set : $a_{0}=\frac{a_{1}^{2}}{32}, \quad a_{2}=8, \quad \lambda=\frac{a_{1}}{8}, \quad \mu=\frac{a_{1}^{2}}{256}, \quad c=0$,

Second solution set : $\quad a_{0}=1+\frac{a_{1}^{2}}{32}, \quad a_{2}=8, \quad \lambda=\frac{a_{1}}{8}, \quad \mu=\frac{64+a_{1}^{2}}{256}, \quad c=1$,

Third solution set : $a_{0}=\frac{-64+a_{1}^{2}}{32}, \quad a_{2}=8, \quad \lambda=\frac{a_{1}}{8}, \quad \mu=\frac{-64+a_{1}^{2}}{256}, \quad c=2$.

Substituting the solutions (10)-(12) into (7), we obtain the hyperbolic function traveling wave solutions

$$
u_{1}(x, t)=2\left[-1+\left(\frac{C_{1} \cosh \frac{1}{2}(x-2 t)+C_{2} \sinh \frac{1}{2}(x-2 t)}{C_{1} \sinh \frac{1}{2}(x-2 t)+C_{2} \cosh \frac{1}{2}(x-2 t)}\right)^{2}\right],
$$

where $C_{1}$ and $C_{2}$ are arbitrary constants; the trigonometric function traveling wave solutions

$$
u_{2}(x, t)=1+2\left(\frac{-C_{1} \sin \frac{1}{2}(x-t)+C_{2} \cos \frac{1}{2}(x-t)}{C_{1} \cos \frac{1}{2}(x-t)+C_{2} \sin \frac{1}{2}(x-t)}\right)^{2},
$$

where $C_{1}$ and $C_{2}$ are arbitrary constants; the rational function solutions

$$
u_{3}(x, t)=\frac{8 C_{2}^{2}}{\left(C_{1}+C_{2} x\right)^{2}},
$$

where $C_{1}$ and $C_{2}$ are arbitrary constants.

In particular, if we take $C_{2} \neq 0, C_{1}^{2}<C_{2}^{2}$ above, then the solutions (13) and (14) become

$$
\begin{array}{ll}
u_{1}(x, t)=-2 \operatorname{sech}\left(\frac{1}{2}(x-2 t)+\xi_{0}\right), & \xi_{0}=\tanh ^{-1}\left(\frac{C_{1}}{C_{2}}\right), \\
u_{2}(x, t)=1+2 \cot ^{2}\left(\frac{1}{2}(x-t)+\xi_{0}\right), & \xi_{0}=\tan ^{-1}\left(\frac{C_{1}}{C_{2}}\right) .
\end{array}
$$

Further, taking $\xi_{0}=0$ in (16) and (17) recovers the bell-shaped solitary solutions and the periodic solutions obtained in $[11,12]$. The rational function solution (15) has not been reported previously to the best of our knowledge.

\subsection{The $2 D-K d V B$ equation}

A second instructive model is the following 2D-KdVB equation in the form:

$$
\left(u_{t}+u u_{x}+\alpha u_{x x x}-\beta u_{x x}\right)_{x}+\gamma u_{y y}=0,
$$


where $\alpha, \beta$, and $\gamma$ are nonzero arbitrary constants. Eq. (18) models waves of fluid in an elastic tube, liquid with small bubbles and turbulance, see [14-20] and references therein. Letting $u(x, y, t)=U(\xi), \xi=x+l y-c t$ in Eq. (18), after integrating the obtained ODE twice, setting the integration constant to zero, we get

$$
\alpha U^{\prime \prime}-\beta U^{\prime}+\frac{1}{2} U^{2}+\left(\gamma l^{2}-c\right) U=0 .
$$

Balancing the terms $U^{\prime \prime}$ and $U^{2}$ in Eq. (19) yields the leading order $N=2$. Therefore, we can write the solution of Eq. (19) in the form (7). Then, substituting (7) and its derivatives (8) and (9) into Eq. (19), and setting the coefficients of $\left(G^{\prime}(\xi) /\right.$ $G(\xi))^{i}(i=0,1, \ldots, 4)$ to zero, we obtain the following under-determined system of algebraic equations for $a_{0}, a_{1}, a_{2}, l, c, \lambda$ and $\mu$ :

$$
\begin{aligned}
& \left(\frac{G^{\prime}}{G}\right)^{0}:-c a_{0}+l^{2} \gamma a_{0}+\frac{a_{0}^{2}}{2}+\beta \mu a_{1}+\alpha \lambda \mu a_{1}+2 \alpha \mu^{2} a_{2}=0 \\
& \left(\frac{G^{\prime}}{G}\right)^{1}:-c a_{1}+l^{2} \gamma a_{1}+\beta \lambda a_{1}+\alpha \lambda^{2} a_{1}+2 \alpha \mu a_{1}+a_{0} a_{1}+2 \beta \mu a_{2}+6 \alpha \lambda \mu a_{2}=0, \\
& \left(\frac{G^{\prime}}{G}\right)^{2}: \beta a_{1}+3 \alpha \lambda a_{1}+\frac{a_{1}^{2}}{2}-c a_{2}+l^{2} \gamma a_{2}+2 \beta \lambda a_{2}+4 \alpha \lambda^{2} a_{2}+8 \alpha \mu a_{2}+a_{0} a_{2}=0, \\
& \left(\frac{G^{\prime}}{G}\right)^{3}: 2 \alpha a_{1}+2 \beta a_{2}+10 \alpha \lambda a_{2}+a_{1} a_{2}=0, \\
& \left(\frac{G^{\prime}}{G}\right)^{4}: 6 \alpha a_{2}+\frac{a_{2}^{2}}{2}=0 .
\end{aligned}
$$

Solving the above system, we have the following two sets of solutions:

First solution set : $a_{0}=-\frac{a_{1}^{2}}{48 \alpha}, \quad a_{2}=-12 \alpha, \quad \lambda=\frac{-12 \beta-5 a_{1}}{60 \alpha}, \quad \mu=\frac{24 \beta a_{1}+5 a_{1}^{2}}{2880 \alpha^{2}}, \quad c=\frac{-6 \beta^{2}}{25 \alpha}+l^{2} \gamma$,

Second solution set : $\quad a_{0}=\frac{576 \beta^{2}-25 a_{1}^{2}}{1200 \alpha}, \quad a_{2}=-12 \alpha, \quad \lambda=\frac{-12 \beta-5 a_{1}}{60 \alpha}, \quad \mu=\frac{24 \beta a_{1}+5 a_{1}^{2}}{2880 \alpha^{2}}, \quad c=\frac{6 \beta^{2}}{25 \alpha}+l^{2} \gamma$.

Substituting the solutions (20) into (7), we obtain the hyperbolic function traveling wave solutions

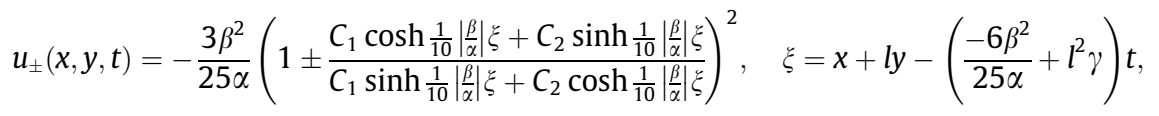

where $\alpha \beta>0$ in the solution function $u_{+}(x, y, t), \alpha \beta<0$ in the solution function $u_{-}(x, y, t), C_{1}$ and $C_{2}$ are arbitrary constants. Also, substituting the solutions (21) into (7), we obtain the hyperbolic function traveling wave solutions

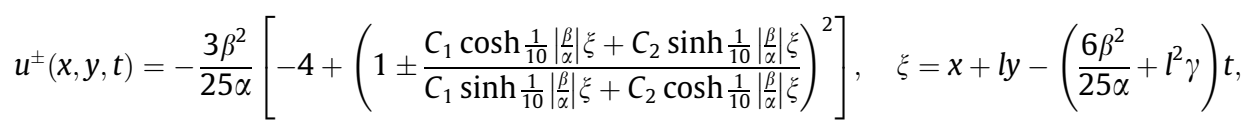

where $\alpha \beta>0$ in the solution function $u^{+}(x, y, t), \alpha \beta<0$ in the solution function $u^{-}(x, y, t), C_{1}$ and $C_{2}$ are arbitrary constants.

In particular, if we take $C_{2} \neq 0, C_{1}^{2}<C_{2}^{2}$ above, then the solution functions become

$$
\begin{aligned}
& u_{ \pm}(x, y, t)=-\frac{3 \beta^{2}}{25 \alpha}\left(1 \pm \tanh \left(\frac{1}{10}\left|\frac{\beta}{\alpha}\right| \xi+\xi_{0}\right)\right)^{2}, \quad \xi=x+l y-\left(\frac{-6 \beta^{2}}{25 \alpha}+l^{2} \gamma\right) t \\
& u^{ \pm}(x, y, t)=-\frac{3 \beta^{2}}{25 \alpha}\left[-4+\left(1 \pm \tanh \left(\frac{1}{10}\left|\frac{\beta}{\alpha}\right| \xi+\xi_{0}\right)\right)^{2}\right], \quad \xi=x+l y-\left(\frac{6 \beta^{2}}{25 \alpha}+l^{2} \gamma\right) t
\end{aligned}
$$

where $\xi_{0}=\tanh ^{-1}\left(\frac{c_{1}}{c_{2}}\right)$. Further, taking $\xi_{0}=0$ in (22) and (23), the solitary solutions obtained in [17] can be recovered by the solution functions $u_{+}(x, y, t)$ and $u^{+}(x, y, t)$.

\section{Conclusion}

In this paper, an implementation of the $\left(G^{\prime} / G\right)$-expansion method is given by applying it to two nonlinear equations to illustrate the validity and advantages of the method. The exact traveling wave solutions being determined in this study are more general, and it is not difficult to arrive at some known analytic solutions for certain choices of the parameters $C_{1}$ and $C_{2}$. Compared with the methods used in [1-6], one can see that the $\left(G^{\prime} / G\right)$-expansion method is not only simple and straightforward, but also avoids tedious calculations. This verifies that the method can be used for many other nonlinear evolution equations. 


\section{References}

[1] J. Weiss, M. Tabor, G. Carnevale, The Painleve property for partial differential equations, J. Math. Phys. 24 (1983) $522-526$.

[2] G.T. Liu, T.Y. Fan, New applications of developed Jacobi elliptic function expansion methods, Phys. Lett. A 345 (2005) $161-166$.

[3] R. Hirota, The Direct Method in Soliton Theory, Cambridge University Press, Cambridge, 2004.

[4] A.M. Wazwaz, Distinct variants of the KdV equation with compact and noncompact structures, Appl. Math. Comput. 150 (2004) $365-377$.

[5] E.G. Fan, Extended tanh-function method and its applications to nonlinear equations, Phys. Lett. A 277 (2000) $212-218$.

[6] J.H. He, X.H. Wu, Exp-function method for nonlinear wave equations, Chaos Solitons Fract. 30 (2006) $700-708$.

[7] M. Wang, X. Li, J. Zhang, The $\left(G^{\prime} / G\right)$-expansion method and traveling wave solutions of nonlinear evolution equations in mathematical physics, Phys. Lett. A 372 (2008) 417-423.

[8] A. Bekir, Application of the $\left(G^{\prime} / G\right)$-expansion method for nonlinear evolution equations, Phys. Lett. A 372 (2008) $3400-3406$.

[9] J. Zhang, X. Wei, Y. Lu, A generalized $\left(G^{\prime} / G\right)$-expansion method and its applications, Phys. Lett. A 372 (2008) 3653-3658.

[10] S. Zhang, J.L. Tong, W. Wang, A generalized ( $\left.G^{\prime} / G\right)$-expansion method for the mKdV equation with variable coefficients, Phys. Lett. A 372 (2008) $2254-$ 2257.

[11] A.M. Wazwaz, Solitary wave solutions for modified forms of Degasperis-Procesi and Camassa-Holm equations, Phys. Lett. A 352 (2006) 500-504.

[12] A.M. Wazwaz, New solitary wave solutions to the modified forms of Degasperis-Procesi and Camassa-Holm equations, Appl. Math. Comput. 186 (2007) 130-141.

[13] E. Yusufoglu, New solitonary solutions for modified forms of DP and CH equations using Exp-function method, Chaos Solitons Fract. doi:10.1016/ j.chaos.2007.07.009.

[14] E. Fan, Extended tanh-function method and its applications to nonlinear equations, Phys. Lett. A 277 (2000) $212-218$.

[15] A. Molabahrami, F. Khani, S.H. Nezhad, Soliton solutions of the two-dimensional KdV-Burgers equation by homotopy perturbation method, Phys. Lett. A 370 (2007) 433-436.

[16] D. Kaya, An application of the decomposition method for the two-dimensional KdV-Burgers equation, Comput. Math. Appl. 48 (2004) $1659-1665$.

[17] E.J. Parkes, Exact solutions to two-dimensional Korteweg-de-Vries-Burgers equation, J. Phys. A: Math. Gen. 27 (1994) L497-L501.

[18] E. Fan, J. Zhang, B.Y.C. Hon, A new complex line soliton for the two-dimensional KdV-Burgers equation, Phys. Lett. A 29 (2001) $376-380$.

[19] Z. Feng, X. Wang, The first integral method to the two-dimensional Burgers-Korteweg-de-Vries equation, Phys. Lett. A 308 (2003) $173-178$.

[20] W. Ma, An exact solution to two-dimensional Korteweg-de-Vries-Burgers equation, J. Phys. A: Math. Gen. 26 (1993) L17-L20. 